\title{
Conectando puntos y atravesando líneas: una aproximación a la investigación artística de NoDOS(3)
}

\section{Connecting points and crossing lines: an approach to the artistic research of NoDOS(3)}

TIPO DE TRABAJO: Comunicación.

PALABRAS CLAVE

Arte, performance, tecnología, espacio, sonido, colaboración.

KEY WORDS

Art, performance, technology, space, sound, collaboration.

RESUMEN

No(DOS)3 es un equipo de trabajo formado por Cristina Ghetti, Julio Sosa y Elia Torrecilla. Tres puntos que conforman una red que conecta e interrelaciona tres maneras de hacer, uniendo intereses y combinando experiencias para crear un nuevo lenguaje basado en lo transmedia. Desde diferentes ámbitos como la pintura, los nuevos medios y la performance, se abordan cuestiones como la línea, el sonido y el cuerpo respectivamente, para investigar y experimentar nuevas relaciones con el espacio urbano, físico y virtual.

En esta comunicación se propone realizar un repaso por una práctica artística colaborativa basada en una puesta en escena que comprende el uso de la tecnología de código abierto, que es activada por unos cuerpos "en acción" con el objetivo de investigar las relaciones que entre ellos se establecen, haciéndolas visibles -y audibles- en el espacio urbano.

La fusión de los intereses y de las diferentes maneras de trabajar de cada uno de los miembros del grupo, permite crear nuevos códigos producto de tres experiencias puestas en común. Son en las diferencias donde nace la posibilidad de crear unos espacios y unos tiempos en los que uno logra desprenderse de sus hábitos para dar rienda suelta al dejarse llevar creativo, surgiendo, hasta ahora, un total de 9 acciones que son aquí revisadas con el objetivo de aproximarnos a las posibilidades creativas y analíticas que ofrece una investigación artística basada en el trabajo colaborativo y la puesta en común.

\section{ABSTRACT}

No(DOS)3 is a work team composed by Cristina Ghetti, Julio Sosa and Elia Torrecilla. Three points that make up a network that connects and interrelates three ways of doing, uniting interests and combining experiences to create a new language based on transmedia. From different areas such as painting, new media and performance, issues such as line, sound and body are addressed, respectively, to investigate and experience new relationships with the urban, physical and virtual space.

This paper proposes a review of a collaborative artistic practice based on a staging that includes the use of open source technology, which is activated by bodies "in action" in order to investigate the relationships between them they are established, making them visible - and audible - in the urban space.

The fusion of the interests and the different ways of working of each one of the members of the group, allows to create new imaginaries product of three experiences put in common. They are in the differences where the possibility of creating spaces and times in which one manages to get rid of their habits to give free rein to get carried away creative, arising, so far, a total of 9 actions that are reviewed here with the objective to approach the creative and analytical possibilities offered by an artistic research based on collaborative work and sharing. 


\section{INTRODUCCIÓN}

NoDOS(3) nace en 2016 por iniciativa de Cristina Ghetti, quien con motivo de su exposición Stripe Generators, realizada en Galería Punto (Valencia, 2016), contacta con Elia Torrecilla para plantear una performance dentro del contexto de su exposición y con Julio Sosa para aportar elementos sonoros a la misma. Tras una primera reunión, surge la manera de unir tres disciplinas (pintura, performance y sonido) a través de la línea, que se establece como el elemento clave de sus acciones. Partiendo de una de las obras que formaban parte de la exposición de Cristina Ghetti, se propone una expansión de la pintura a través de la línea, que por un lado es extendida por Elia Torrecilla y Cristina Ghetti desde la propia pieza hasta la calle; por otro lado, también se extiende a través del sonido que toma la forma de ondas sonoras básicas (sinusoidales, cuadradas y dientes de sierra). Estas líneas sonoras son introducidas por Julio Sosa en los teléfonos móviles que las performers llevan adheridos a sus cuerpos y que con sus movimientos activan y modifican el sonido en tiempo real.

Tras esta primera experiencia y hasta la actualidad, $\operatorname{NoDOS(3)~ha~realizado~un~total~de~nueve~acciones~(numeradas~en~serie:~Acción\# 1,~}$ Acción\#2, Acción\#3...) en las que exploran a través de sus cuerpos esos espacios híbridos que se sitúan entre lo físico y lo virtual y aquellos espacios intermedios entre el interior y el exterior, captando, a través de los dispositivos tecnológicos, las diferentes ondas sónicas que se emiten en conexión con el espacio atravesado. En acciones como la \#3 y la \#7, el sonido se encuentra vinculado a las coordenadas espaciales pertenecientes a un recorrido previamente señalado, activándose en tiempo real a través de la relación directa entre cuerpo y espacio y modulándose a partir de los movimientos realizados. En todas sus acciones, presentan una actitud exploratoria como si fueran seres recién aterrizados de otro planeta que aparecen, con sus trajes y prótesis y con una estética, también híbrida, que va evolucionando en cada acción, incorporando luces, antenas y diferentes materiales que parecen dialogar con un tiempo pasado desde una visión futurista.

\section{PROCESO}

Como sostiene Teresa Marín (2007), la creación colectiva conlleva un proceso de desmitificación del mito del genio que obliga a modificar algunas estrategias creativas y desarrollar otras nuevas. Se trata de "un conjunto de procesos que permiten realizar una actividad creativa y alcanzar un objetivo común entre varios individuos, que siendo diferentes, comparten motivaciones y experiencias, con independencia de la forma de organización y relación que se establezca entre ellos".

Esto es algo en lo que hallamos puntos positivos y negativos: el trabajo en grupo nos permite intercambiar ideas, generar un ambiente de libertad creativa y experimental, unir intereses, descubrir otras maneras de crear, aprender nuevas disciplinas y observar desde otros puntos de vista una misma cosa. En definitiva, compartir, aprender y divertirse en la práctica artística.

Sin embargo, en momentos en los que debemos tomar decisiones, en ocasiones se producen cortocircuitos, y cuando se trabaja bajo la presión de una fecha límite, no siempre es fácil organizarse, pues por un lado cada uno tiene sus horarios y ocupaciones, y por otro, en las fases de planteamiento de los proyectos, existen bloqueos, desacuerdos e inseguridades que tienen lugar a la hora de tomar decisiones. Sin embargo estos aspectos del trabajo grupal considerados como negativos, son transformados en positivos ya que cada miembro del grupo aporta sus habilidades y lenguajes propios, dando lugar a interesantes diálogos creativos. Cuando en una etapa del proyecto, algún componente del grupo se muestra inseguro o poco participativo, al llegar a otra fase, ese comportamiento de transforma en una energía que logra impulsar al resto. Así logramos alcanzar un equilibrio, y aquello que en un momento dado supone una dificultad, se convierte en estímulo.

El trabajo en grupo nos permite en este caso, trabajar con un modelo más próximo al rol del "artista productor", que al del "artista creador", implicándonos en la gestión, en un proceso más proyectual y de posproducción que en la propia materialización y ejecución directa del trabajo (Marín, 2007); de hecho, nuestras acciones podrían ser consideradas procesos basados en la experimentación, el azar y el error como estrategia.

De este modo, nuestro proceso creativo se hace pues visible -y audible- en las acciones que se presentan en público, donde tratamos de promover unas experiencias compartidas basadas en la participación y el contacto humano, conscientes de que el arte, funciona en este sentido como un elemento mediador entre sujetos y espacios. En este sentido, comprendemos el dispositivo tecnológico (teléfono móvil), no como reflejo, sino como antena amplificadora, tomando la metáfora que McLuhan desarrolló del artista como la "antena de la carrera" (McLuhan, 1964), explicando que este es un experto que comprende los cambios en la percepción de los sentidos, por ello hace uso de la tecnología de una manera libre, anticipándose a su impacto transformador y siendo consciente del reto cultural y tecnológico que ello supone. Para McLuhan, los medios de expresión como el arte o la poesía son capaces de abrir 
caminos para comprender en mayor profundidad las 'acciones' o los patrones de lo instrumental y las formas en que estructuran nuestra propia existencia.

Lo pulido, lo liso, lo impecable, son la seña de identidad de nuestra época. Son lo que tiene en común las esculturas de Jeff Koons, los smartphones y la depilación. Estas cualidades ponen en evidencia el "exceso de positividad" que Han desarrolla en el campo del arte y de la estética. Porque hoy en día nos gusta tanto lo pulido? Se pregunta Han. Porque no daña, no ofrece resistencia. Lo bello digital constituye un espacio liso y pulido de lo igual, un espacio que no tolera ninguna extrañeza, ninguna alteridad, ninguna negatividad (Chul Han, 2015).

\section{RESULTADOS}

Mediante el empleo de dispositivos móviles, que utilizamos expresamente como extensiones de nuestros cuerpos (McLuhan, 1996) hacemos visible el hecho de que es el cuerpo el que actúa como el motor que hace posible que la tecnología sea móvil, y por tanto generador de historias.

A través de los movimientos que realizamos en el espacio, se convierten en actos trazables gracias al uso de los medios locativos (De Souza e Silva, 2006), unos trazos visuales y sonoros para ser recorridos a través de diferentes interfaces en tiempo real. En este sentido, la práctica artística de NoDOS(3) propone una experiencia de la interfaz como umbral, como puerta de acceso, como una vía de comunicación con el lenguaje-máquina de los dispositivos digitales.

En este conjunto de acciones el empleo de la tecnología nos permite obtener una experiencia más plurisensorial y más afectiva (apegada) con el espacio que nos rodea (Torrecilla, 2018). Las nuevas ventanas que nos han aportado las tecnologías de la comunicación nos abocan al conocimiento inmediato y completo de los acontecimientos con la sensación de estar "dentro de la Historia", pero sin controlarla.

Durante la edición 2017 del Congreso Arte Ciencia Ciudad, Rogelio López Cuenca ejemplificaba con el caso de la ciudad de Málaga, las transformaciones que sufren los espacios urbanos. Las políticas neoliberales someten al centro urbano a una radical reducción y especialización funcional que lo convierte en un "parque temático". El esquema se repite en diversas ciudades; el entorno pasa rápidamente de ser un espacio habitado a un espacio visitable, consumible, utilizado como atractivo turístico.

Mientras que la multiplicidad y simultaneidad de actividades propias de la ciudad y sus habitantes son expulsadas a los márgenes, el antiguo centro histórico, en nombre de su conversión en ciudad-museo se transforma en un centro comercial abierto, con un abrumador predominio de la hostelería. Todo va a girar en torno a la satisfacción de las necesidades y expectativas del turista. En los centros histórico, donde la población residente sufre una caída vertiginosa, las actividades se ven limitadas prácticamente a dos: circular y consumir. Pero las prácticas culturales y artísticas pueden constituir también un espacio en litigio, un territorio desde el que es posible cuestionar el discurso dominante y las representaciones hegemónicas, si dotamos a las ciudades de un trabajo que reflexione en el ámbito de lo artístico y de lo común entendiendo la ciudad como un organismo vivo y vibrante capaz de generar distintas capas de percepción.

La estructura, las relaciones, las de poder y las otras, los equipamientos, el trabajo, la vida doméstica, la diversidad, el género, la tecnología, la política, la economía y lo social forman parte del entramado de direcciones que se superponen en la aprehensión de la ciudad. Todas estas capas no son sino la capacidad de la que dispone la población para asirse al lugar que habita; en otras palabras, una suerte de cultura colectiva que permite construir discurso de vida o, mejor dicho, de (con)vivencia. La experiencia urbana es el punto de partida sobre el que dibujar el mapa de acontecimientos e interpretaciones que delimitan el significado en sí mismo, así como la imagen mental proyectada y el papel que desempeña el espacio en las relaciones que se establecen entre individuos.

\section{DOSSIER (Selección)}

\section{Acción \#1}

NoDOS(3) presenta en Galería Punto, una propuesta ad hoc para la exposición "Stripe-generators" de Cristina Ghetti. La propia artista, junto con Julio Sosa y Elia Torrecilla, plantean su primera acción en conjunto en la que confluyen diferentes intereses. Tomando la línea como protagonista, esta se extiende en el espacio buscando la interacción con el público y la expansión hacia el exterior del espacio expositivo. El desplazamiento producido por el cuerpo en la prolongación de la línea se vuelve sensible a través de dispositivos móviles que traducen el movimiento a un sonido de ondas sinusoidales. 
El componente interactivo/sonoro se basa en una instalación compuesta por teléfonos móviles, software personalizado y un ordenador. Los teléfonos móviles van ajustados a los cuerpos de los performers, recogiendo información del movimiento mediante sensores del mismo dispositivo. Esta información se envía por una red inalámbrica dedicada a un ordenador con un software de procesado de audio, que convierte la información del movimiento a sonido. Esta instalación permite la improvisación en vivo, ya que el sonido se va generando según el movimiento de los intérpretes. Los posibles parámetros del sonido se han mantenido utilizando parámetros estéticos binarios, utilizando dos posibles valores para el sonido, haciendo referencia a las pinturas de Cristina Ghetti de esta exposición, que en su mayoría utilizan una paleta de color binaria.

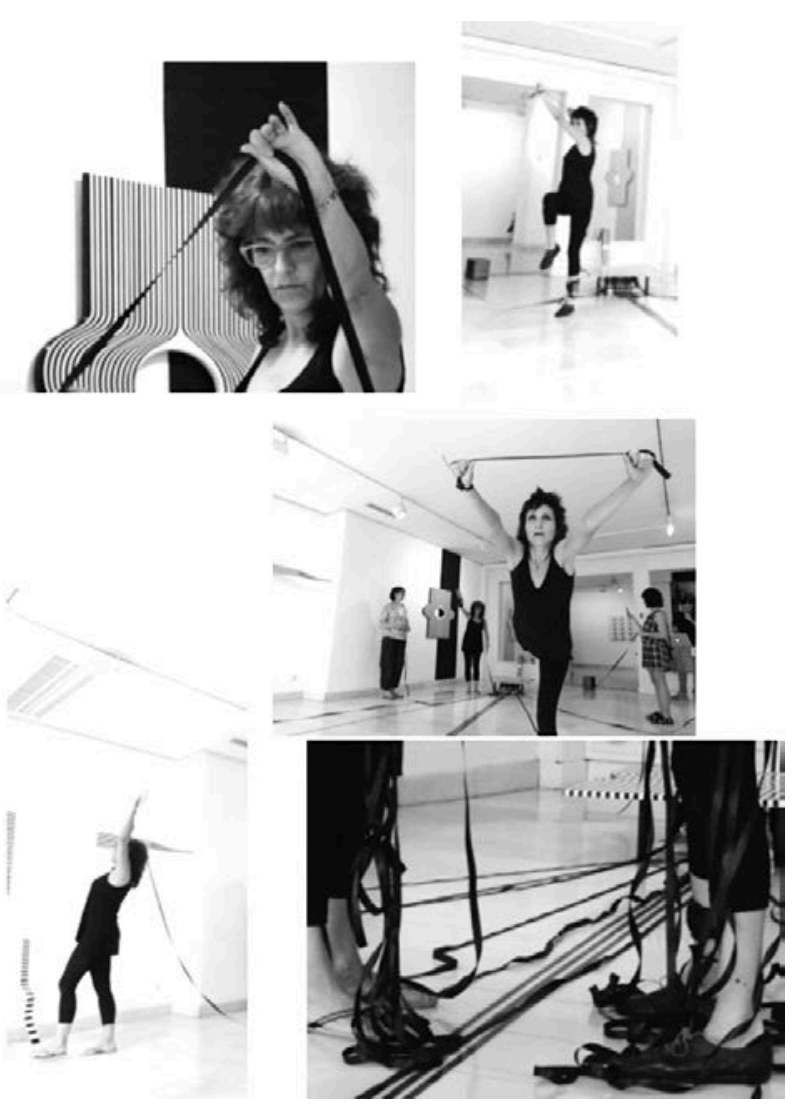

Figura 1. Fotografías correspondientes a Acción\#1 realizada en Galería Punto (Valencia), 2016. Vídeo disponible en: https://vimeo.com/192686040

\section{Acción \#2}

NoDOS(3) presenta su segunda acción que se plantea como un trabajo híbrido, en el que lo virtual y la descorporeización, se vincula al espacio físico a través de la línea pictórica que se desvanece hacia un espacio puramente digital. Un trabajo donde la estética del error informático y la relación del cuerpo con la línea en un espacio bidimensional, adquiere una tercera dimensión gracias a la luz y el sonido en tiempo real. El producto final se puede considerar un videomapping y en su creación se utilizaron diversas técnicas de producción congruentes con nuestros conceptos. Para generar el sonido, se utilizó un sistema similar al de Acción\#1, qué convierte los movimientos de los intérpretes en sonido. En la imagen se utilizaron técnicas y procesos como la sobresaturación, pixelado, excesos de contrastes y repetición. 

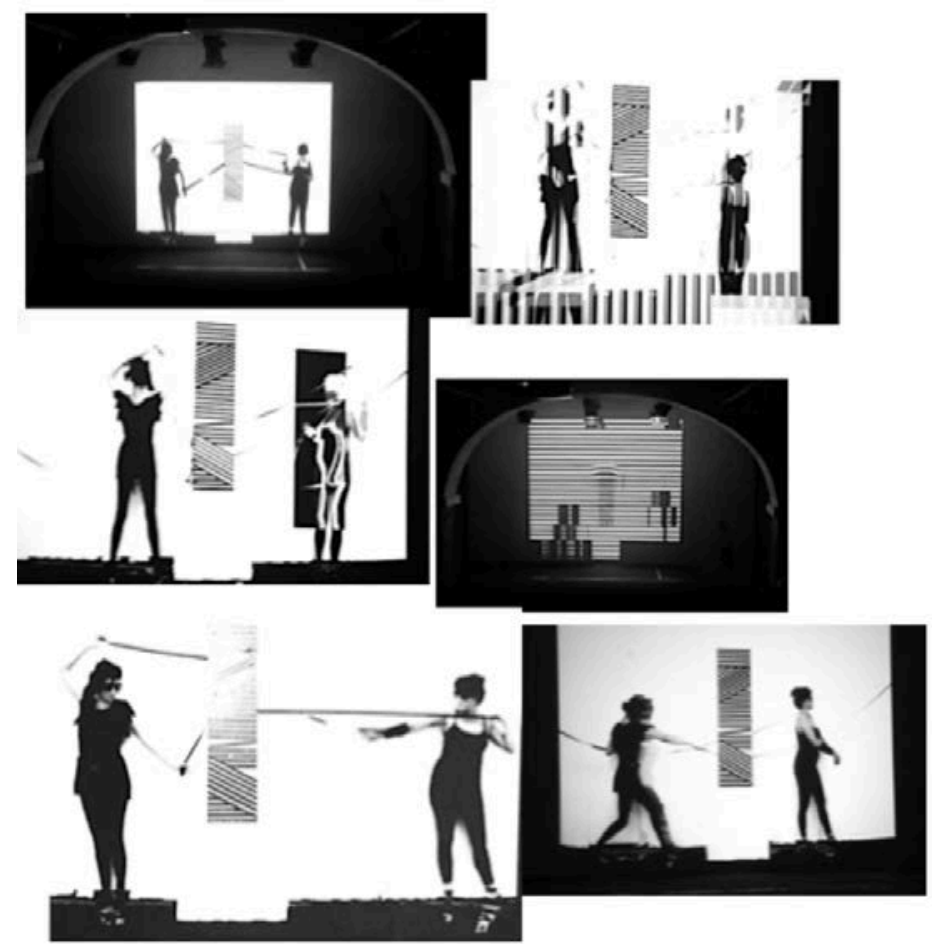

Figura 2. Fotografías correspondientes a Acción\#2 realizada en Ca Revolta dentro de la programación del Festival de Arte Intramurs (Valencia, 2016). Vídeo disponible en: https://vimeo.com/193578872

\section{Acción \#3}

Haciendo uso de los elementos que componen su trabajo (línea - cuerpo - sonido), NoDOS(3) propone una acción en la que se busca una interacción con los habitantes del barrio y un acercamiento del espacio expositivo a la calle. Acción\#3 se desarrolla principalmente en el espacio público planteando, en primer lugar, un recorrido por Belleville en el que se recopilan las coordenadas transitadas (esto es, nuestra posición en el espacio y en el tiempo); este conjunto de datos, es traducido mediante un programa informático, a una serie de ondas sonoras que serán activadas a través de dos dispositivos móviles empleados durante la acción, en la que las performers crearán una red simbólica, haciendo uso de la línea y estableciendo puntos de unión (nodos) entre el espacio urbano, los transeúntes y el espacio expositivo.

Acción \#3 consiste en caminar una línea que, interpretada a través del sonido se vuelve audible, y visible en forma de cintas que establecen vínculos entre los participantes y entre estos y su entorno.

Para la interacción y el sonido en esta acción, nos basamos en el sistema de interacción con dispositivos móviles que utilizamos anteriormente. La gran diferencia es que aquí prescindimos del ordenador y de la red dedicada. Todo el sonido se generaba desde el mismo dispositivo utilizando una aplicación que recogía los datos de los sensores del mismo y que los combinaba con los valores de las coordenadas GPS del recorrido realizado. Esto nos permitió realizar a cabo una acción totalmente móvil e inalámbrica, que no dependía de ningún componente fijo. 


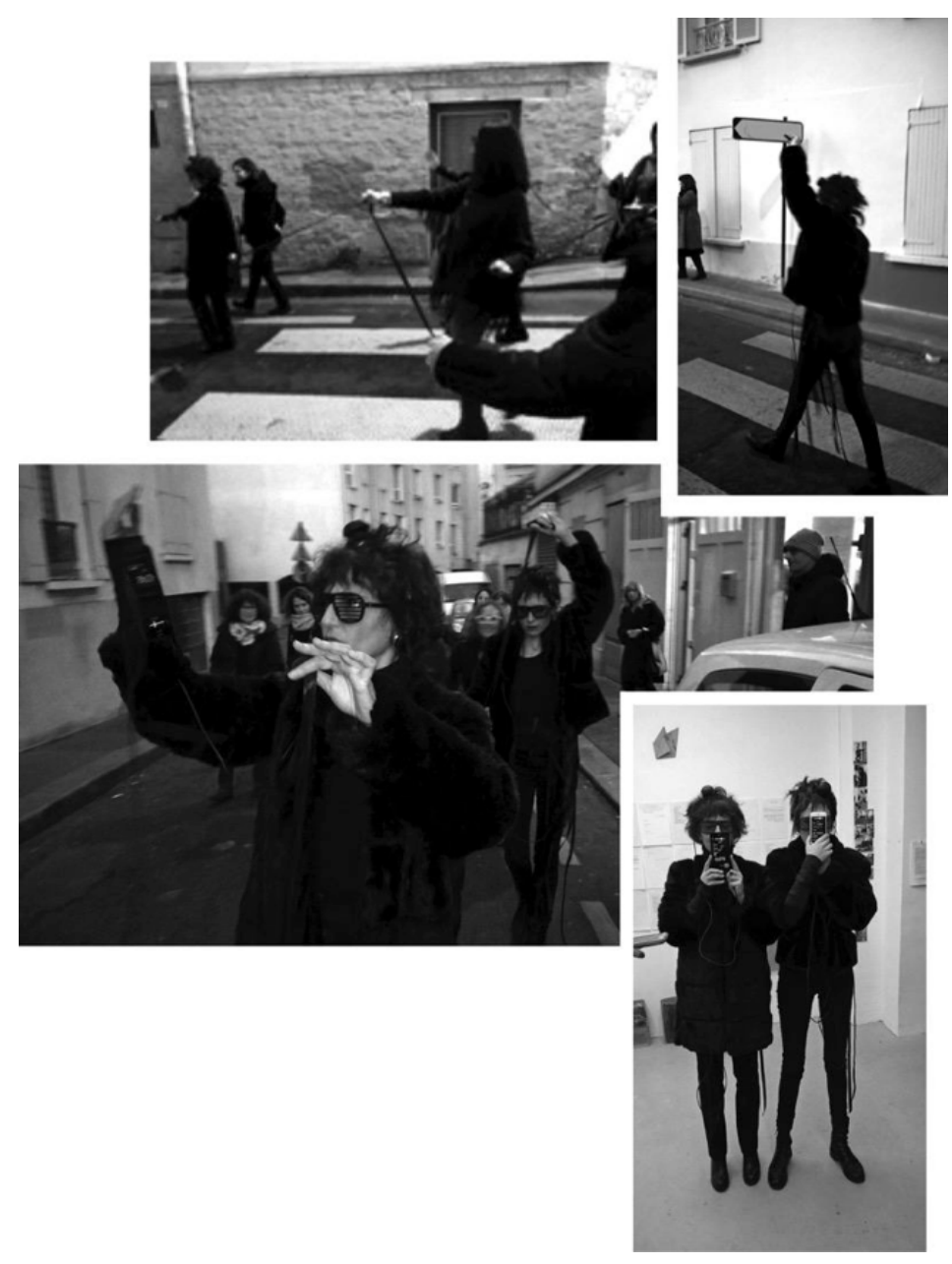

Figura 3. Fotografías correspondientes a Acción\#3 realizada en Espace Julio PopUp (Belleville, París), 2017

\section{Acción \#4}

Para este performance, nos inspiramos en la composición "Les Barricades mysterieuses" de Couperin. Utilizamos elementos del barroco junto con nuestra línea existente de investigación centrada en medios interactivos. Para generar el sonido, utilizamos el sistema de interacción con dispositivos móviles. Como de costumbre, los móviles iban ajustados a los cuerpos de las performers, y con los datos del movimiento, se manipulaba un proceso de síntesis granular sobre una grabación de la pieza de Couperin, lo cual da como resultado una especie de deconstrucción de la composición musical mediante la exploración física y su relación con el espacio. 


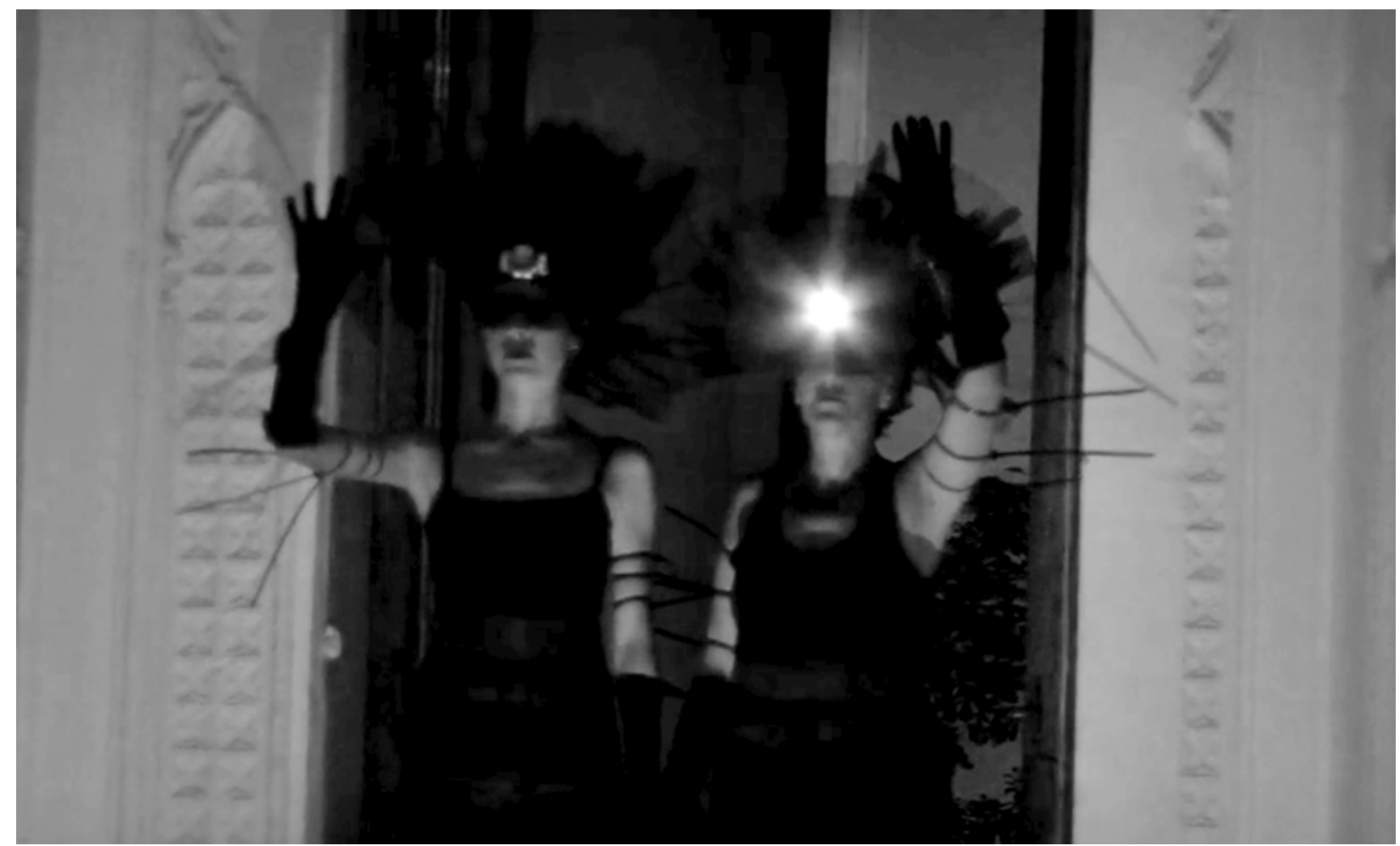

Figura 4. Imagen correspondiente a Acción\#4 realizada la Ermita de Villagadea, sede de la Cátedra Institucional de Estudios Artísticos Anetta Nicoli de la UMH y la Concejalía de Cultura del Ayuntamiento de Altea inauguran el Ciclo de Conciertos La Ruta de les Ermites (Altea, Alicante), 2017.

\section{Acción \#5}

Para la Acción \#5 decidimos crear un sistema en nuestro performance con la intención de reducir nuestra participación a la performance hasta convertirnos en un dispositivo más de toda la instalación multimedia e interactiva. Primero, delimitamos nuestras posibles acciones: las dos performers tenían un rango posible de movimientos dependiendo del sonido que se reprodujera, el cual era controlado por el performer (un comodín en el sistema, ya que controlaba los sonidos a su antojo). Solo había 3 opciones diferentes de sonido: ondas sinusoidales, ondas cuadradas y ruido blanco. El desenlace de la acción consistía en un fallo o descomposición del sistema, donde las performers se rebelan contra estos roles establecidos al inicio.

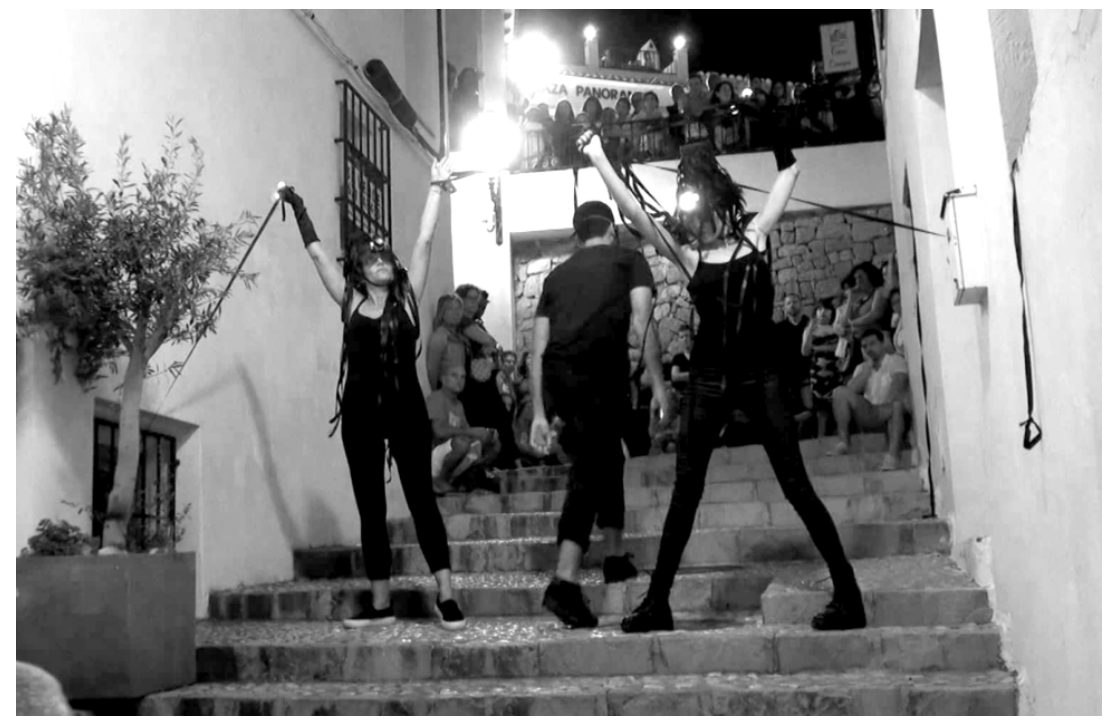

Figura 5. Imagen correspondiente a Acción\#5 realizada en Altea Nit de L'Art 2017 


\section{Acción \#7}

Acción\#7 se realizó en el contexto del Tercer Congreso Internacional ACC: Arte, Ciencia y Ciudad en la Facultad de Bellas Artes de Málaga, planteada como una adaptación de Acción\#3, que busca una interacción entre el espacio institucional (la universidad), la calle y los participantes. Siguiendo el mismo procedimiento, en Acción\#7 se proyectó previamente un recorrido desde el espacio virtual del cual se obtuvieron las coordenadas. Este conjunto de datos numéricos fueron traducidos mediante un programa informático, a una serie de ondas sonoras que fueron activadas en el momento en que atravesamos los puntos exactos pertenecientes a dichas coordenadas. Esta activación sonora del espacio se produjo través de los dos dispositivos adheridos a nuestros cuerpos, esta vez mediante una prótesis que integraba tanto el teléfono móvil como los dos altavoces.

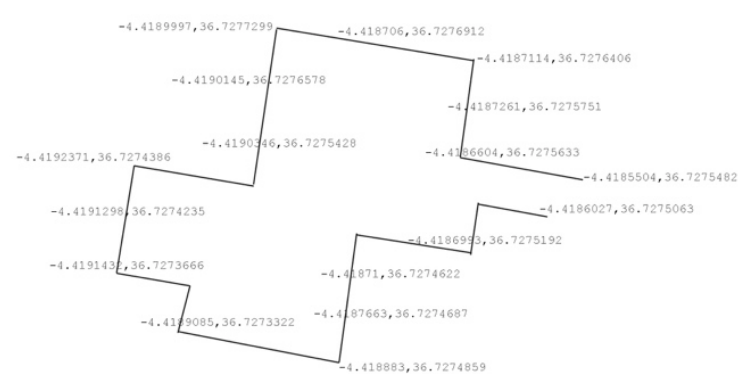

Figuras 6 y 7. Plano del recorrido y muestra de algunas de las coordenadas recopiladas para Acción\#7

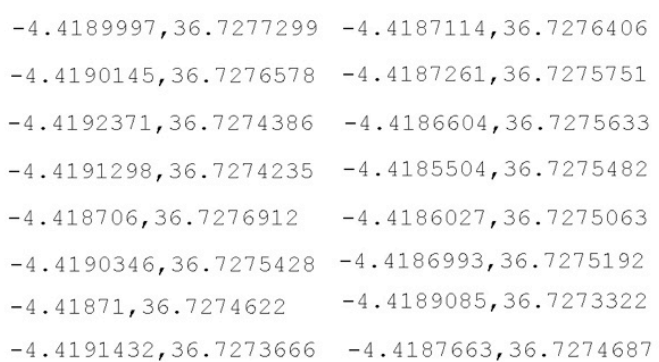

$-4.4191432,36.7273666-4.4187663,36.7274687$

Durante el recorrido, de forma simultánea se produjo una red simbólica en la que extendimos unas líneas (cintas) que nos permitieron interactuar con el público-participante, estableciendo puntos de unión (nodos) entre el espacio urbano (físico y digital) y sus usuarios. Una red de coordenadas situada entre el espacio vivido y el espacio abstracto, dando lugar a una experiencia en un espacio híbrido o en una ciudad-interfaz a través de un cuerpo-software.
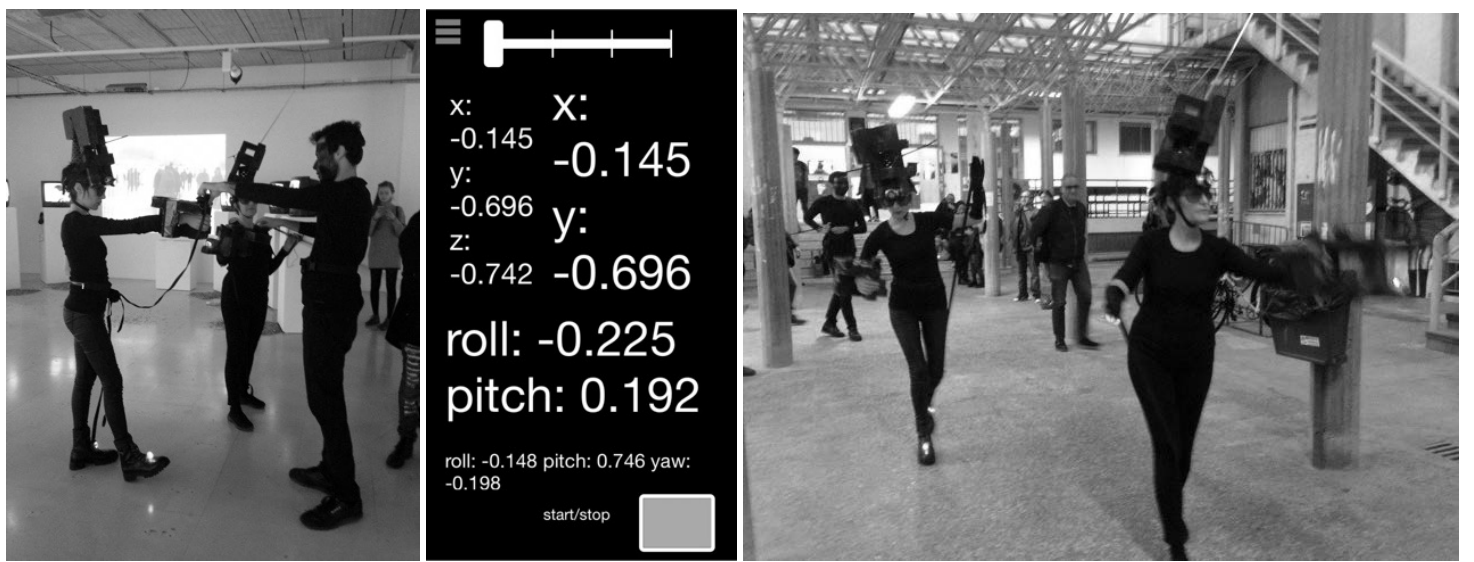

Figuras 8 y 9. Fotografías correspondientes a Acción\#7 realizada en Málaga, 2017 dentro del Tercer Congreso Internacional ACC: Arte, Ciencia y Ciudad, y el diseño de la interfaz empleada durante la acción. 


\section{Acción \#9 “Ráyate”}

Es una pieza de performance en la que se invita a las personas personas de 1-88 años de edad a colocarse una camiseta a rayas y pasar un momento lúdico interactuando con una proyección que muestra líneas en movimiento en un espacio y evolucionando con música en directo. Nos interesa proponer una complicidad que se forme entre los artistas intérpretes y los ejecutantes que se sientan cómodos en su entorno recientemente ocupado, creen sus propios espacios con sus propias reglas para el compromiso y el juego, logrando así una comunidad revitalizada a través de la experiencia compartida. Desde el punto de vista plástico, hacemos que las intenciones de la abstracción y del op-art se expandan, animando la pintura geométrica y creando nuevas configuraciones con cada movimiento personal. El espacio inmersivo, la música y la interacción entre la gente proponen un momento de comunión y juego. Hacemos aquí también referencia a la tradición de explorar la relación entre el cuerpo y el objeto, y mixturando medios, proponemos una performance donde el público será el principal protagonista.

Aquí realizamos una video performance, donde utilizamos un video con música preparado anteriormente. Para continuar con el tema de la línea y la geometría, creamos música utilizando ondas sonoras básicas, como sinusoidales y cuadradas. La tonalidad de los sonidos eran uniformes en casi toda la música, junto con efectos de bucle, efectos panorámicos y de desfase, que juegan con la percepción del espacio y el sonido del público.
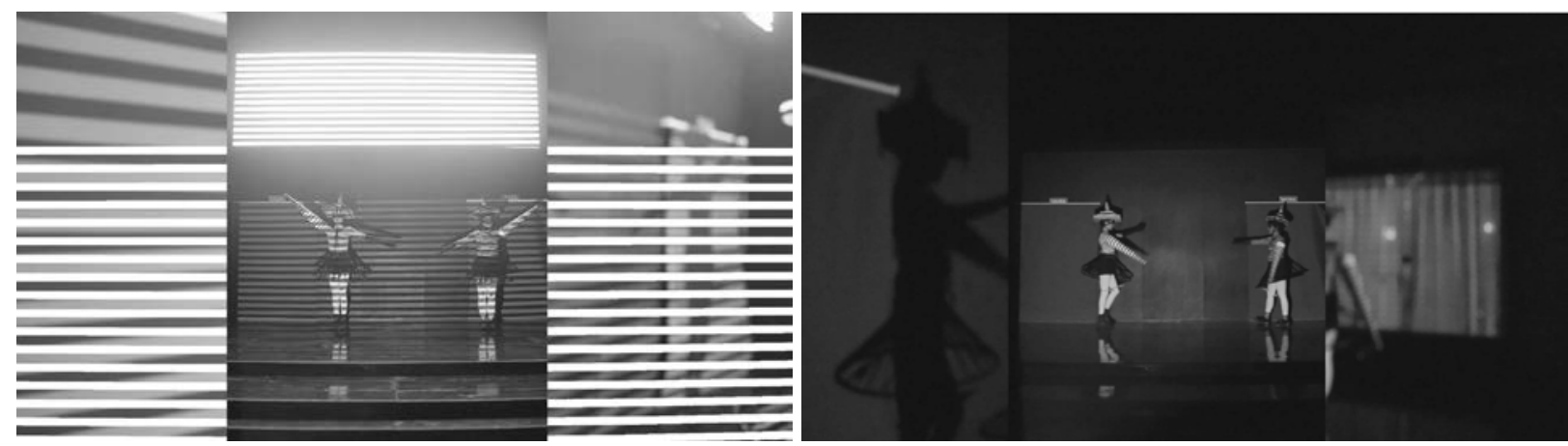

Figuras 10 y 11. Fotografías correspondientes a Acción\#9 "Ráyate" realizada dentro del Festival de Arte Intramurs (Valencia, 2018).

\section{FUENTES REFERENCIALES}

Byung Chul, H. (2015). La salvación de lo bello. Barcelona: Herder Editorial.

De Souza e Silva, A. (2006). From Cyber to Hybrid. Mobile Technologies as Interfaces to Hybrid Spaces. Space and Culture, 9 (3), $261-$ 278.

Marín García, T. (2007). Estrategias de creación colectiva e el arte contemporáneo. En Marín García, T. y Krakowski, A. (coords.) (2007). Tecnologías y estrategias para la creación artística. Elche: Universidad Miguel Hernández-Alfa ediciones gráficas.

McLuhan, M. (1964). Comprender los medios de comunicación. Las extensiones del ser humano. Barcelona: Paidós, 1996.

Torrecilla, E. (2018). El lenguaje de las interfaces en movimiento: La hibridación en la práctica artística de NoDOS (3). ASRI. Arte y Sociedad. Revista de Investigación, (15), 7-14. Disponible en: http://asri.eumed.net/15/lenguaje-interfaces-movimiento.pdf

Torrecilla, E. (2018). Flâneur, Ciberflâneur, Phoneur: Un paseo de resignificación del flâneur por el espacio urbano moderno, el ciberespacio y el espacio híbrido. Acciones artísticas propias entre 2011-2018 [Tesis doctoral]. Universitat Politècnica de València. Disponible en: https://riunet.upv.es/handle/10251/107391 